\title{
Phytocannabis for the treatment of cancer-related or cancer treatment related symptoms: Evidence Based Review
}

\author{
Article by Kavita Gupta ${ }^{1}$ \\ PhD Scholar at Texila American University, Guyana \\ E-mail: 16kavitagupta@texilaconnect.com
}

\begin{abstract}
Cancer is defined as a generic term for a large group of diseases that was observed to affect any part of the body, often characterized by abnormal rapid growth of abnormal cells. According to WHO report, Cancer was the leading cause of morbidity and mortality worldwide. The primary goal of the treatment is to cure cancer or to considerably prolong life, along with improved patient's quality of life by palliative care, psychological support and alternative treatments. The present report focused on the use of Phytocannabis and its derivatives to alleviate the symptoms occurred due to cancer that included reduced appetite, chemotherapy-induced nausea and vomiting, radiotherapy-induced pain, nausea and vomiting in order to attenuate the disease process. Cancer, Chemotherapy and Radiotherapyinduced emesis and pain, all these mentioned factors led to the interrogation and investigation of the anti-emesis, pain relief, and mood stabilizing properties of Phytocannabis. This study presented the update on health and social consequences of Phytocannabis use, with a focus on the long-term and frequent use of medicinal Cannabis and its derivatives in alleviating the cancer related symptoms. It aimed to present the current knowledge on the impact of Phytocannabis use on health, from its impact on treating cancer related symptoms to its role in chemotherapy and Radiotherapy induced symptoms. This report evaluated the evidence on whether long-term Phytocannabis use is a contributory cause of the following health outcomes: relief from pain, nausea, vomiting, appetite, food taste, night sweats, and adverse physical and mental health effects such as mood swings, fatigue, hallucinations, postural hypotension, dizziness, mind alertness. Thus, the present paper reported of the use of Phytocannabis and its derivatives such as, Nabilone, Delta-9THC, and Cannabis available in different forms (Oral, Inhaled, Sublingual) on the Quality of life of cancer patients who underwent Chemotherapy treatment and Radiotherapy treatment.
\end{abstract}

Keywords: 'cannabis', 'marijuana', 'cannabinoids', 'tetrahydrocannabinol', 'THC', 'dronabinol', 'cannabidiol', 'CBD', 'cannabidivarin', 'nabilone', 'CBDV', 'cancer', 'chemotherapy', 'radiotherapy' ,'nausea' 'vomiting', 'pain', 'open-label studies', Randomized controlled trials'.

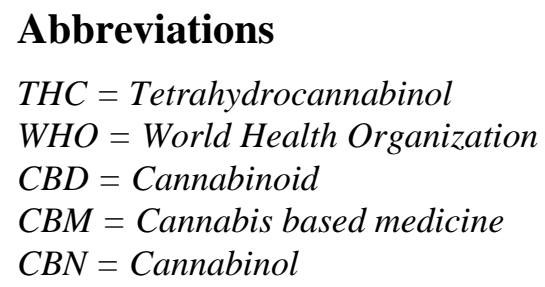

\section{Introduction}

Cancer today has become the leading cause of death worldwide, which accounted for approximately 8.2 million deaths in the year 2012[1]. According to WHO survey report, the most common cause of cancer deaths were observed for lung cancer (1.59 million deaths), liver cancer (745 000 deaths), stomach cancer (723 000 deaths), colorectal cancer (694 000 deaths), breast cancer (521 000 deaths), esophageal cancer (400 000 deaths) [1]. Epidemiologically, it was observed that approximately more than $60 \%$ of the world's total 
new annual cases along with $70 \%$ of the world's cancer deaths occured in Africa, Asia and Central and South America [1]. This disease burden had led to the invention of various effective conventional treatments [1]. However, there were very few of the treatments found to be effective in improving the quality of life of cancer patients undergoing different regimen of treatments[1]. Therefore, the present paper highlighted the use of Phytocannabis as an alternative treatment to alleviate the side effects produced in the treatment of cancer[1]. Phytocannabinoids weredefined as the cannabinoid compounds that were obtained from the femaleCannabis sativa or Cannabis indicaplant which wasfound to act on cannabinoid receptors in cells that modulated neurotransmitter release in the brain[1, 2]. The principal cannabinoids in the cannabis plant included delta-9-tetrahydrocannabinol (THC), cannabidiol (CBD), and cannabinol (CBN)[1, 2]. The known chemical composition of Cannabis sativa kept constantly changing[2]. From 2005 to 2015, the number of cannabinoids identified in the whole plant increased from 70 to 104 and other known compounds in the plant increased from some 400 to around 650[2]. Nevertheless, there were some relatively good data on the prevalence of Phytocannabis in some parts of the world[2]. Levels of lifetime use differ considerably between countries, ranging from around one third of adults in Denmark, France and the United Kingdom, to 8\% or less than 1 in 10 in Bulgaria, Romania and Turkey [2].

\section{Aim}

The main objective of this review was:

a. To understand the role of Phytocannabis and its derivatives in the treatment of cancer associated symptoms, like pain, nausea and vomiting,

b. To assess clinical safety and efficacy of Phytocannabis in reducing accompanied symptoms due to result of cancer, Chemotherapy or Radiotherapy treatment,

c. To evaluate the potential therapeutic value of Phytocannabis in preventing cancerous tumor growth, as well as, alleviating and reduction in pain, nausea and vomiting.

d. To collect more data on the increasing use of PHYTOCANNABIS in CANCER.

\section{Search methodology}

The primary and foremost point that was focused throughout the article review was 'Cannabis and Cancer'. All types of studies were included in this regard. Moreover, children, Adolescents and Adults suffering from Cancer were included in this review. The methodology being adopted for the relevant literature review, searching the databases like, PUBMED, Cochrane Library, MEDLINE, EMBASE, Clinical trials.gov by using keywords such as, 'cannabis', 'marijuana', 'cannabinoids', 'tetrahydrocannabinol', 'THC', 'cannabidiol', 'CBD', 'cannabidivarin', 'CBDV', 'cancer', 'nausea' and 'vomiting' was utilized. Moreover, a greater number of google searches were done to gather reliable and valid information from websites primarily focusing on safety and efficacy of medicinal cannabis used in cancer patients. The search was confined to studies being published in English language. Moreover, PRISMA methodology was adopted for inclusion of relevant studies.

\section{Prisma flowchart methodology}


South American Journal of Clinical Research

Volume 3, Issue 1, 2016

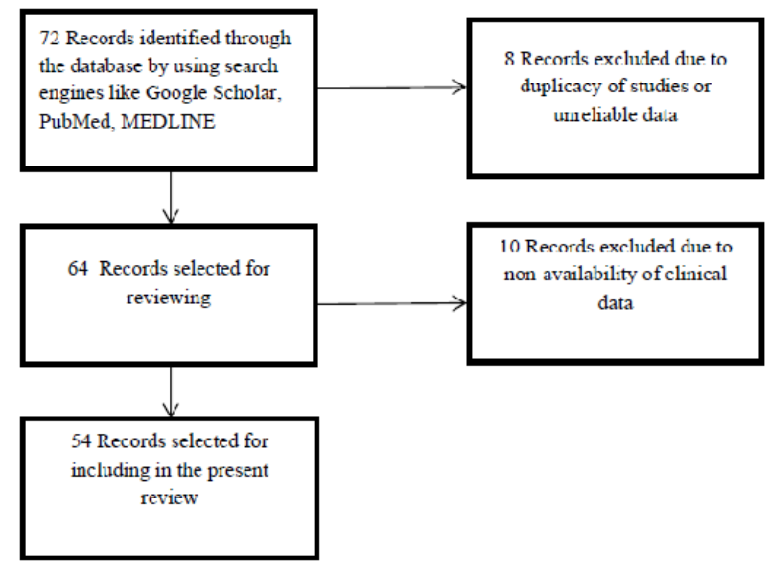

Fig 1: PRISMA FLOWCHART For Selection of Clinical Studies. 
South American Journal of Clinical Research

Volume 3, Issue 1, 2016

Description of Randomized Controlled Clinical Studies

\begin{tabular}{|c|c|c|c|c|c|c|c|c|c|c|}
\hline S.No. & Study & $\begin{array}{l}\text { Indicatio } \\
\text { n }\end{array}$ & $\begin{array}{l}\text { Populatio } \\
\text { n }\end{array}$ & $\begin{array}{l}\text { Study } \\
\text { Design }\end{array}$ & $\begin{array}{l}\text { Intervention } \\
+ \\
\text { Route of } \\
\text { Administrati } \\
\text { on }\end{array}$ & Intervention & Control & Results & $\begin{array}{l}\text { Adverse } \\
\text { Events }\end{array}$ & Significance \\
\hline 1. & $\begin{array}{l}\text { Côté, M. } \\
\text { et al. } \\
2015 \\
\text { [3]. }\end{array}$ & Cancer & $\begin{array}{l}66 \\
\text { patients } \\
\text { with } \\
\text { cancer } \\
\text { undergoin } \\
\text { g } \\
\text { Radiother } \\
\text { apy }\end{array}$ & $\begin{array}{l}\text { Controlled } \\
\text { study }\end{array}$ & $\begin{array}{l}\text { Nabilone } \\
\text { Orally }\end{array}$ & Nabilone & Placebo & $\begin{array}{l}\text { Pain, nausea } \\
\text { and loss of } \\
\text { appetite } \\
\text { persisted }\end{array}$ & $\begin{array}{l}\text { Weight } \\
\text { reduction and } \\
\text { abrupted sleep }\end{array}$ & $\begin{array}{l}\text { Efficacy not } \\
\text { obtained, no } \\
\text { significant } \\
\text { results }\end{array}$ \\
\hline 2. & $\begin{array}{l}\text { Lynch, } \\
\text { M.E. et } \\
\text { al. } \\
2014 \\
{[4] .}\end{array}$ & Cancer & $\begin{array}{l}16 \\
\text { patients } \\
\text { with } \\
\text { chemother } \\
\text { apy- } \\
\text { induced } \\
\text { neuropathi } \\
\text { c pain } \\
\end{array}$ & $\begin{array}{l}\text { Controlled } \\
\text { study, } \\
\text { cross-over } \\
\text { study }\end{array}$ & $\begin{array}{l}\text { Cannabis } \\
\text { Sublingually }\end{array}$ & $\begin{array}{l}\text { cannabinoid } \\
\text { agent, } \\
\text { nabiximols } \\
\text { (oral mucosal } \\
\text { spray } \\
\text { containing } \\
\text { cannabinoids)+ } \\
\text { Chemotherapy } \\
\end{array}$ & Placebo & $\begin{array}{l}\text { Greater } \\
\text { reduction in } \\
\text { pain }\end{array}$ & $\begin{array}{l}\text { Nausea and } \\
\text { loss of appetite }\end{array}$ & $\begin{array}{l}\text { Reduction in } \\
\text { pain intensity }\end{array}$ \\
\hline 3. & $\begin{array}{l}\text { Portenoy, } \\
\text { R.K. et } \\
\text { al. } \\
2012 \\
{[5] .}\end{array}$ & Cancer & $\begin{array}{l}263 \\
\text { patients } \\
\text { with } \\
\text { advanced } \\
\text { cancer }\end{array}$ & $\begin{array}{l}\text { Controlled } \\
\text { study }\end{array}$ & $\begin{array}{l}\text { Cannabis } \\
\text { Sublingually }\end{array}$ & $\begin{array}{l}\text { nabiximols at a } \\
\text { low dose (1-4 } \\
\text { sprays/day), } \\
\text { medium dose } \\
\text { (6-10 } \\
\text { sprays/day), or } \\
\text { high dose (11- } \\
16 \text { sprays/day) }\end{array}$ & Placebo & $\begin{array}{l}\text { Reduction in } \\
\text { pain }\end{array}$ & $\begin{array}{l}\text { Dose related } \\
\text { side effects }\end{array}$ & $\begin{array}{l}\text { Could be } \\
\text { considered as } \\
\text { an add-on } \\
\text { Therapy for } \\
\text { additional } \\
\text { pain } \\
\text { reduction }\end{array}$ \\
\hline 4. & Brisbois, & Cancer & 46 & Controlled & Delta-9-THC & THC (2.5 mg, n & placebo & THC & None & useful in the \\
\hline
\end{tabular}


South American Journal of Clinical Research

Volume 3, Issue 1, 2016

\begin{tabular}{|c|c|c|c|c|c|c|c|c|c|c|}
\hline & $\begin{array}{l}\text { T.D. et } \\
\text { al. } \\
2011 \\
{[6] .}\end{array}$ & & $\begin{array}{l}\text { patients } \\
\text { with } \\
\text { advanced } \\
\text { cancer }\end{array}$ & study & Orally & $=24)$ & $\begin{array}{l}\text { oral } \\
\text { capsules (n } \\
=22 \text { ) twice } \\
\text { daily for } 18 \\
\text { days }\end{array}$ & $\begin{array}{l}\text { improved } \\
\text { taste and } \\
\text { appetite }\end{array}$ & & $\begin{array}{l}\text { palliation of } \\
\text { chemosensor } \\
\text { y alterations } \\
\text { and improved } \\
\text { food taste }\end{array}$ \\
\hline 5. & $\begin{array}{l}\text { Duran, } \\
\text { M. et al. } \\
2010 \\
{[7] .}\end{array}$ & Cancer & $\begin{array}{l}16 \\
\text { patients of } \\
\text { chemother } \\
\text { apy- } \\
\text { induced } \\
\text { nausea }\end{array}$ & $\begin{array}{l}\text { Controlled } \\
\text { study }\end{array}$ & $\begin{array}{l}\text { Cannabis } \\
\text { Sublingually }\end{array}$ & $\begin{array}{l}\text { cannabis-based } \\
\text { medicine } \\
\text { (CBM) } \\
\text { containing } \\
\text { delta-9- } \\
\text { tetrahydrocann } \\
\text { abinol and } \\
\text { cannabidiol }\end{array}$ & $\begin{array}{l}\text { Placebo } \\
\text { (standard } \\
\text { anti-emetic } \\
\text { treatment) }\end{array}$ & $\begin{array}{l}\text { Reduction in } \\
\text { nausea and } \\
\text { vomiting }\end{array}$ & $\begin{array}{l}\text { no relevant } \\
\text { side-effects }\end{array}$ & $\begin{array}{l}\text { Well } \\
\text { tolerated } \\
\text { drug }\end{array}$ \\
\hline 6. & $\begin{array}{l}\text { Johnson, } \\
\text { J.R. et al. } \\
2010 \\
{[8] .}\end{array}$ & Cancer & $\begin{array}{l}177 \\
\text { cancer } \\
\text { patients } \\
\text { with pain }\end{array}$ & $\begin{array}{l}\text { Controlled } \\
\text { study }\end{array}$ & $\begin{array}{l}\text { Cannabis + } \\
\text { Delta-9-THC } \\
\text { extract } \\
\text { Sublingually }\end{array}$ & $\begin{array}{l}\text { THC:CBD } \\
\text { extract }(n= \\
60), \text { THC } \\
\text { extract } \\
(n=58)\end{array}$ & $\begin{array}{l}\text { Placebo } \\
(\mathrm{n}=59)\end{array}$ & $\begin{array}{l}\text { Improved } \\
\text { sleep quality, } \\
\text { reduced pain } \\
\text { and nausea }\end{array}$ & $\begin{array}{l}\text { drug-related } \\
\text { adverse events }\end{array}$ & $\begin{array}{l}\text { A cannabis } \\
\text { extract } \\
\text { containing } \\
\text { THC:CBD } \\
\text { was superior } \\
\text { in reducing } \\
\text { pain } \\
\end{array}$ \\
\hline 8. & $\begin{array}{l}\text { Strasser, } \\
\text { F. et al. } \\
2006 \\
{[10] .}\end{array}$ & Cancer & $\begin{array}{l}243 \\
\text { cancer } \\
\text { patients } \\
\text { with } \\
\text { weight }\end{array}$ & $\begin{array}{l}\text { Controlled } \\
\text { study }\end{array}$ & $\begin{array}{l}\text { Cannabis + } \\
\text { Delta-9-THC } \\
\text { extract orally }\end{array}$ & $\begin{array}{l}\text { Cannabis } \\
\text { Extract n= } 95 \\
\text { (standardized } \\
\text { for } 2.5 \mathrm{mg} \\
\text { THC and } 1 \mathrm{mg}\end{array}$ & $\begin{array}{l}\text { Placebo } \\
(n=48)\end{array}$ & $\begin{array}{l}\text { Increased } \\
\text { appetite }\end{array}$ & $\begin{array}{l}\text { no relevant } \\
\text { side-effects }\end{array}$ & $\begin{array}{l}\text { Well } \\
\text { tolerated }\end{array}$ \\
\hline
\end{tabular}


South American Journal of Clinical Research

Volume 3, Issue 1, 2016

\begin{tabular}{|c|c|c|c|c|c|c|c|c|c|c|}
\hline & & & loss & & & $\begin{array}{l}\text { cannabidiol) or } \\
\text { THC ( } 2.5 \mathrm{mg}) \\
\mathrm{n}=100\end{array}$ & & & & \\
\hline 9. & $\begin{array}{l}\text { Jatoi, A. } \\
\text { et al. } \\
2002 \\
{[11] .}\end{array}$ & Cancer & $\begin{array}{l}469 \\
\text { cancer } \\
\text { patients }\end{array}$ & $\begin{array}{l}\text { Controlled } \\
\text { study }\end{array}$ & $\begin{array}{l}\text { Delta-9-THC } \\
\text { Orally }\end{array}$ & $\begin{array}{l}\text { 1. oral } \\
\text { megestrol } \\
\text { acetate } 800 \\
\text { mg/d liquid } \\
\text { suspension + } \\
\text { placebo, } \\
\text { 2. oral } \\
\text { dronabinol } 2.5 \\
\text { mg twice a day } \\
+ \text { placebo, or } \\
\text { 3. both agents }\end{array}$ & placebo & $\begin{array}{l}\text { Improved } \\
\text { appetite }\end{array}$ & $\begin{array}{l}\text { no relevant } \\
\text { side-effects }\end{array}$ & $\begin{array}{l}\text { Combination } \\
\text { therapy not } \\
\text { effective }\end{array}$ \\
\hline 10. & $\begin{array}{l}\text { Lane, M. } \\
\text { et al. } \\
1991 \\
{[12] .}\end{array}$ & Cancer & $\begin{array}{l}67 \\
\text { patients } \\
\text { on various } \\
\text { cancer } \\
\text { chemother } \\
\text { apy } \\
\text { treatments }\end{array}$ & $\begin{array}{l}\text { Controlled } \\
\text { study }\end{array}$ & $\begin{array}{l}\text { Delta-9-THC } \\
\text { Orally }\end{array}$ & $\begin{array}{l}\text { 1. dronabinol } \\
10 \text { mg every } 6 \\
\text { hr plus } \\
\text { placebo; } \\
\text { 2. placebo plus } \\
\text { prochlorperazin } \\
\text { e } 10 \text { mg every } \\
6 \text { hr; or } \\
\text { 3. dronabinol } \\
\text { and } \\
\text { prochlorperazin } \\
\text { e, each } 10 \text { mg } \\
\text { every } 6 \text { hr }\end{array}$ & placebo & $\begin{array}{l}\text { Prevented } \\
\text { vomiting }\end{array}$ & $\begin{array}{l}\text { no relevant } \\
\text { side-effects }\end{array}$ & $\begin{array}{l}\text { Effective } \\
\text { treatment }\end{array}$ \\
\hline 11. & $\begin{array}{l}\text { McCabe, } \\
\text { M. et al. } \\
1988\end{array}$ & Cancer & $\begin{array}{l}36 \\
\text { patients } \\
\text { with }\end{array}$ & $\begin{array}{l}\text { Controlled } \\
\text { study }\end{array}$ & $\begin{array}{l}\text { Delta-9-THC } \\
\text { Orally }\end{array}$ & $\begin{array}{l}\text { Oral Delta-9- } \\
\text { THC }\end{array}$ & $\begin{array}{l}\text { Placebo } \\
\text { (Prochlorpe } \\
\text { razine) }\end{array}$ & $\begin{array}{l}\text { Reduction in } \\
\text { nausea and } \\
\text { vomiting }\end{array}$ & Dysphoria & $\begin{array}{l}\text { Excellent } \\
\text { antiemetic } \\
\text { control }\end{array}$ \\
\hline
\end{tabular}


South American Journal of Clinical Research Volume 3, Issue 1, 2016

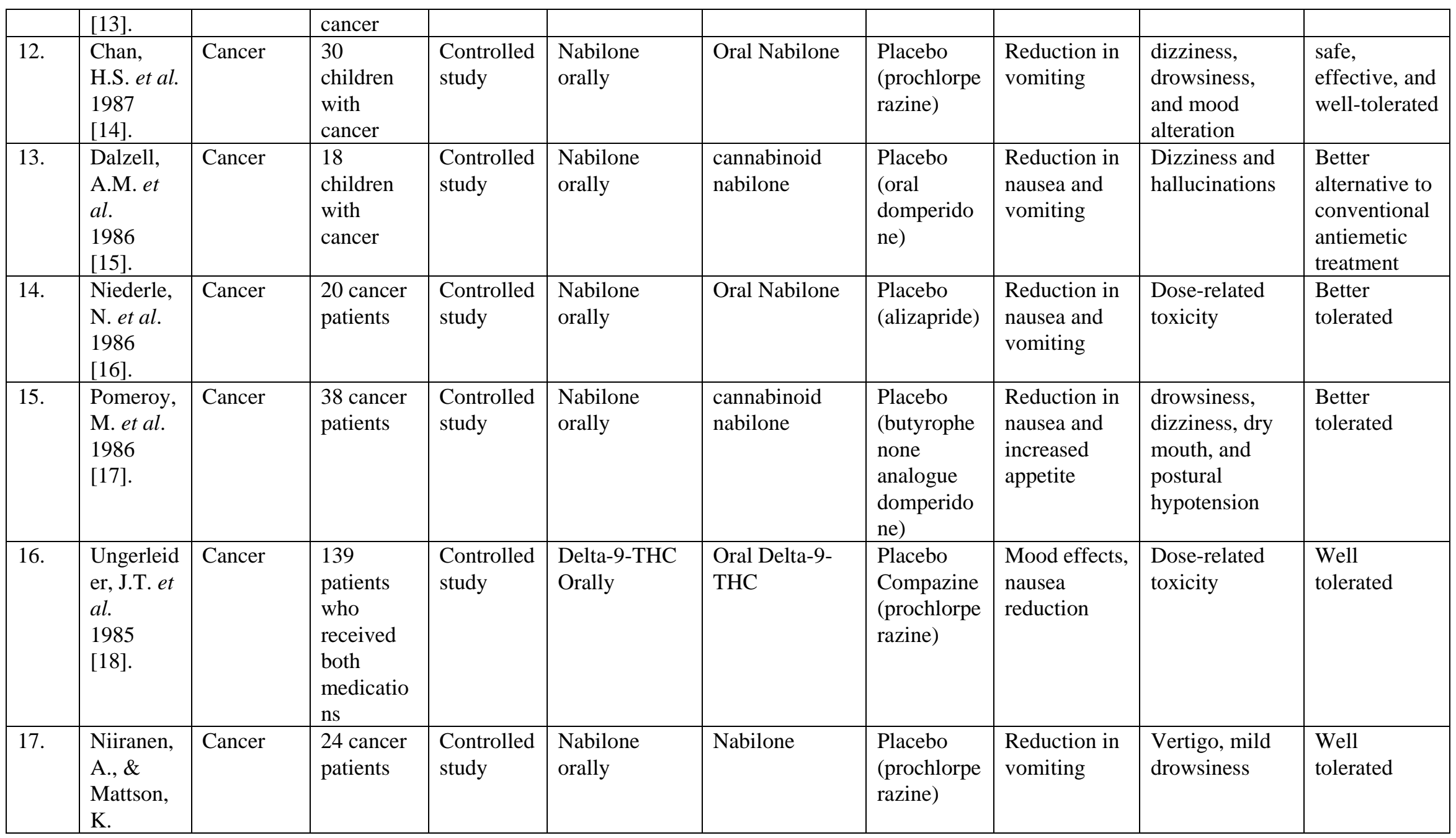


South American Journal of Clinical Research

Volume 3, Issue 1, 2016

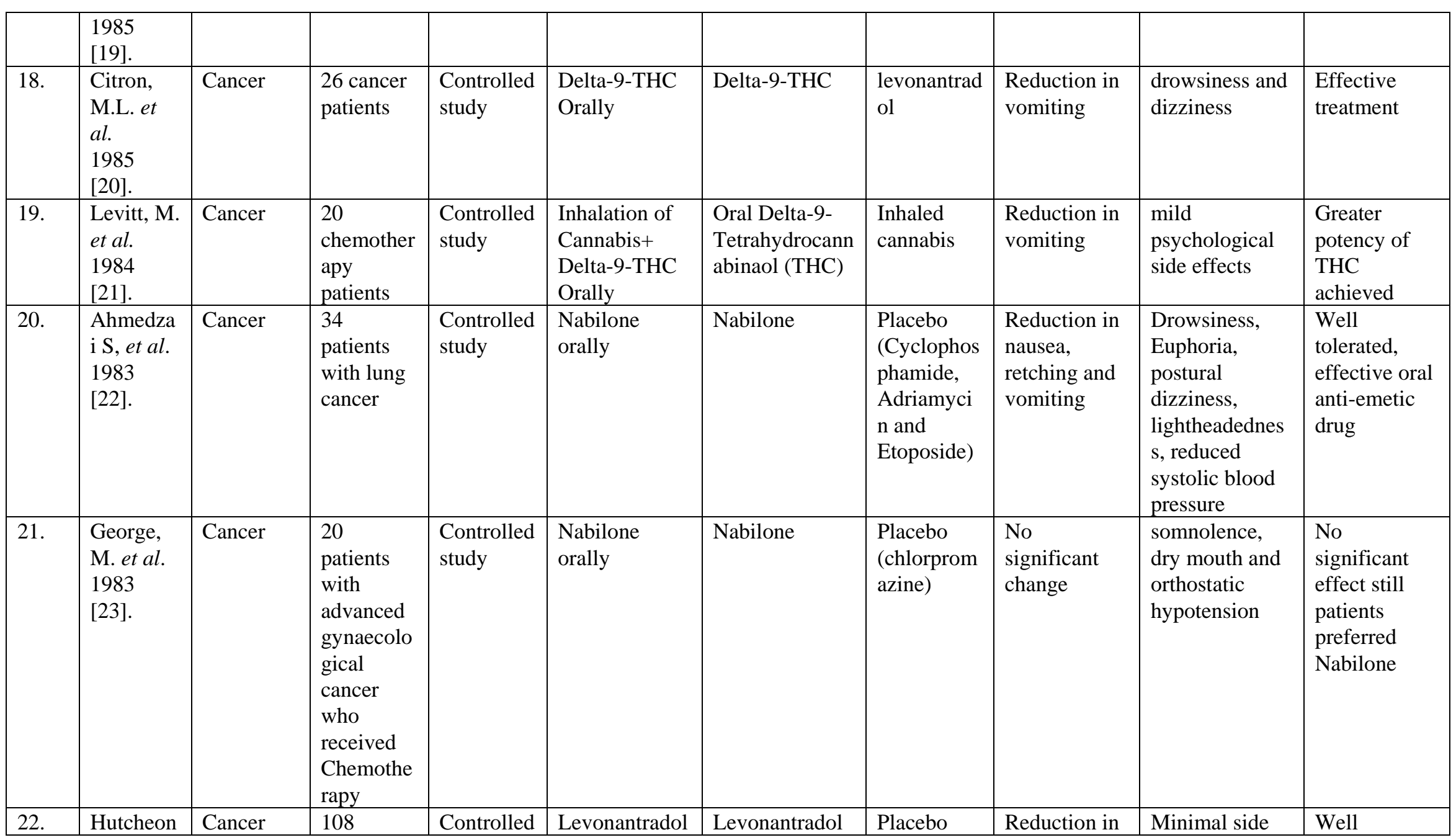


South American Journal of Clinical Research

Volume 3, Issue 1, 2016

\begin{tabular}{|c|c|c|c|c|c|c|c|c|c|c|}
\hline & $\begin{array}{l}\text {, A.W. et } \\
\text { al. } \\
1983 \\
{[24] .}\end{array}$ & & $\begin{array}{l}\text { patients } \\
\text { undergoin } \\
\mathrm{g} \text { cancer } \\
\text { chemother } \\
\text { apy }\end{array}$ & study & orally & cannabinoid & $\begin{array}{l}\text { (chlorprom } \\
\text { azine) }\end{array}$ & vomiting & effects & tolerated \\
\hline 23. & $\begin{array}{l}\text { Ungerleid } \\
\text { er, J.T. et } \\
\text { al. } \\
1982 \\
{[25] .}\end{array}$ & Cancer & $\begin{array}{l}214 \\
\text { cancer } \\
\text { patients } \\
\text { receiving } \\
\text { chemother } \\
\text { apy }\end{array}$ & $\begin{array}{l}\text { Controlled } \\
\text { study }\end{array}$ & $\begin{array}{l}\text { Delta-9-THC } \\
\text { Orally }\end{array}$ & Delta-9-THC & $\begin{array}{l}\text { Placebo } \\
\text { (prochlorpe } \\
\text { razine) }\end{array}$ & $\begin{array}{l}\text { Reduction in } \\
\text { nausea and } \\
\text { vomiting }\end{array}$ & $\begin{array}{l}\text { less ability to } \\
\text { concentrate, } \\
\text { less social } \\
\text { interaction, and } \\
\text { less activity }\end{array}$ & $\begin{array}{l}\text { Well } \\
\text { tolerated }\end{array}$ \\
\hline 24. & $\begin{array}{l}\text { Johansso } \\
\text { n, R. et } \\
\text { al. } \\
1982 \\
\text { [26]. }\end{array}$ & Cancer & $\begin{array}{l}27 \\
\text { patients } \\
\text { on } \\
\text { chemother } \\
\text { apy }\end{array}$ & $\begin{array}{l}\text { Controlled } \\
\text { study }\end{array}$ & $\begin{array}{l}\text { Nabilone } \\
\text { orally }\end{array}$ & Nabilone & $\begin{array}{l}\text { Placebo } \\
\text { (prochlorpe } \\
\text { razine) }\end{array}$ & $\begin{array}{l}\text { Reduction in } \\
\text { nausea, } \\
\text { vomiting and } \\
\text { dry retching } \\
\text { episodes }\end{array}$ & $\begin{array}{l}\text { Postural } \\
\text { hypotension, } \\
\text { Vertigo, } \\
\text { headache, } \\
\text { depression, } \\
\text { general } \\
\text { weakness, } \\
\text { mood } \\
\text { alterations with } \\
\text { dysphoria }\end{array}$ & $\begin{array}{l}\text { Well } \\
\text { tolerated }\end{array}$ \\
\hline
\end{tabular}


South American Journal of Clinical Research

Volume 3, Issue 1, 2016

\begin{tabular}{|c|c|c|c|c|c|c|c|c|c|c|}
\hline 26. & $\begin{array}{l}\text { Chang, } \\
\text { A.E. et } \\
\text { al. } \\
1981 \\
\text { [28]. }\end{array}$ & Cancer & $\begin{array}{l}8 \text { patients } \\
\text { on } \\
\text { chemother } \\
\text { apy }\end{array}$ & $\begin{array}{l}\text { Controlled } \\
\text { study }\end{array}$ & $\begin{array}{l}\text { oral and } \\
\text { inhaled delta- } \\
\text { 9- } \\
\text { tetrahydrocan } \\
\text { nabinol (THC) }\end{array}$ & $\begin{array}{l}\text { oral and } \\
\text { smoked delta- } \\
9 \text { - } \\
\text { tetrahydrocann } \\
\text { abinol (THC) }\end{array}$ & $\begin{array}{l}\text { Placebo } \\
\text { (Adriamyci } \\
\mathrm{n} \text { and } \\
\text { Cytoxan } \\
\text { chemothera } \\
\text { py) }\end{array}$ & $\begin{array}{l}\text { No } \\
\text { significant } \\
\text { effect on } \\
\text { nausea and } \\
\text { vomiting } \\
\text { produced }\end{array}$ & Minimal & $\begin{array}{l}\text { No } \\
\text { significant } \\
\text { change }\end{array}$ \\
\hline 27. & $\begin{array}{l}\text { Neidhart, } \\
\text { J.A. et al. } \\
1981 \\
{[29] .}\end{array}$ & Cancer & $\begin{array}{l}52 \\
\text { patients } \\
\text { with } \\
\text { cancer } \\
\text { chemother } \\
\text { apy }\end{array}$ & $\begin{array}{l}\text { Controlled } \\
\text { study }\end{array}$ & $\begin{array}{l}\text { Delta-9-THC } \\
\text { orally }\end{array}$ & Delta-9-THC & $\begin{array}{l}\text { Placebo } \\
\text { (haloperido } \\
\text { l) }\end{array}$ & $\begin{array}{l}\text { Reduction in } \\
\text { nausea and } \\
\text { vomiting }\end{array}$ & $\begin{array}{l}\text { no serious side } \\
\text { effects }\end{array}$ & $\begin{array}{l}\text { Well } \\
\text { tolerated }\end{array}$ \\
\hline 28. & $\begin{array}{l}\text { Einhorn, } \\
\text { L.H. et } \\
\text { al. } \\
1981 \\
{[30] .}\end{array}$ & Cancer & $\begin{array}{l}85 \\
\text { patients } \\
\text { receiving } \\
\text { chemother } \\
\text { apy }\end{array}$ & $\begin{array}{l}\text { Controlled } \\
\text { study }\end{array}$ & $\begin{array}{l}\text { Nabilone } \\
\text { orally }\end{array}$ & Oral Nabilone & $\begin{array}{l}\text { Placebo } \\
\text { (prochlorpe } \\
\text { razine) }\end{array}$ & $\begin{array}{l}\text { Reduction in } \\
\text { nausea and } \\
\text { vomiting }\end{array}$ & $\begin{array}{l}\text { hypotension } \\
\text { and lethargy }\end{array}$ & $\begin{array}{l}\text { Well } \\
\text { tolerated }\end{array}$ \\
\hline 29. & $\begin{array}{l}\text { Sallan, } \\
\text { S.E. et al. } \\
1980 \\
{[31] .}\end{array}$ & Cancer & $\begin{array}{l}20 \text { cancer } \\
\text { patients }\end{array}$ & $\begin{array}{l}\text { Controlled } \\
\text { study }\end{array}$ & $\begin{array}{l}\text { Delta-9-THC } \\
\text { orally }\end{array}$ & $\begin{array}{l}\text { Oral Delta-9- } \\
\text { THC }\end{array}$ & $\begin{array}{l}\text { Placebo } \\
\text { (prochlorpe } \\
\text { razine) }\end{array}$ & $\begin{array}{l}\text { Reduction in } \\
\text { nausea and } \\
\text { vomiting and } \\
\text { improved } \\
\text { appetite }\end{array}$ & minimal & $\begin{array}{l}\text { Well } \\
\text { tolerated }\end{array}$ \\
\hline 30. & $\begin{array}{l}\text { Orr, L.E. } \\
\text { et al. } \\
1980 \\
{[32] .}\end{array}$ & Cancer & $\begin{array}{l}55 \text { cancer } \\
\text { patients }\end{array}$ & $\begin{array}{l}\text { Controlled } \\
\text { study }\end{array}$ & $\begin{array}{l}\text { Delta-9-THC } \\
\text { orally }\end{array}$ & $\begin{array}{l}\text { Oral Delta-9- } \\
\text { THC }\end{array}$ & $\begin{array}{l}\text { Placebo } \\
\text { (prochlorpe } \\
\text { razine) }\end{array}$ & $\begin{array}{l}\text { Reduction in } \\
\text { nausea and } \\
\text { vomiting and } \\
\text { improved } \\
\text { appetite }\end{array}$ & $\begin{array}{l}\text { no serious side } \\
\text { effects }\end{array}$ & $\begin{array}{l}\text { Well } \\
\text { tolerated }\end{array}$ \\
\hline 31. & $\begin{array}{l}\text { Steele, N. } \\
\text { et al. } \\
1980 \\
{[33] .}\end{array}$ & Cancer & $\begin{array}{l}37 \\
\text { patients } \\
\text { on } \\
\text { chemother }\end{array}$ & $\begin{array}{l}\text { Controlled } \\
\text { study }\end{array}$ & $\begin{array}{l}\text { Nabilone } \\
\text { orally }\end{array}$ & Oral Nabilone & $\begin{array}{l}\text { Placebo } \\
\text { (prochlorpe } \\
\text { razine) }\end{array}$ & $\begin{array}{l}\text { Reduction in } \\
\text { vomiting }\end{array}$ & $\begin{array}{l}\text { Mild } \\
\text { drowsiness and } \\
\text { dizziness }\end{array}$ & $\begin{array}{l}\text { Well } \\
\text { tolerated }\end{array}$ \\
\hline
\end{tabular}


South American Journal of Clinical Research Volume 3, Issue 1, 2016

\begin{tabular}{|c|c|c|c|c|c|c|c|c|c|c|}
\hline & & & apy & & & & & & & \\
\hline 35. & $\begin{array}{l}\text { Sallan, } \\
\text { S.E. et al. } \\
1975 \\
{[37] .}\end{array}$ & Cancer & $\begin{array}{l}84 \text { cancer } \\
\text { patients }\end{array}$ & $\begin{array}{l}\text { Controlled } \\
\text { study }\end{array}$ & $\begin{array}{l}\text { Delta-9-THC } \\
\text { orally }\end{array}$ & $\begin{array}{l}\text { Oral Delta-9- } \\
\text { THC }\end{array}$ & Placebo & $\begin{array}{l}\text { Reduction in } \\
\text { vomiting }\end{array}$ & $\begin{array}{l}\text { Sedation and } \\
\text { mental } \\
\text { clouding }\end{array}$ & $\begin{array}{l}\text { Well } \\
\text { tolerated }\end{array}$ \\
\hline 37. & $\begin{array}{l}\text { Noyes, R. } \\
\text { Jr et al. } \\
1975 \\
\text { [39]. }\end{array}$ & Cancer & $\begin{array}{l}36 \text { cancer } \\
\text { patients }\end{array}$ & $\begin{array}{l}\text { Controlled } \\
\text { study }\end{array}$ & $\begin{array}{l}\text { Delta-9-THC } \\
\text { orally }\end{array}$ & $\begin{array}{l}\text { Oral Delta-9- } \\
\text { THC }\end{array}$ & Placebo & $\begin{array}{l}\text { Reduction in } \\
\text { pain }\end{array}$ & $\begin{array}{l}\text { somnolence, } \\
\text { dizziness, } \\
\text { ataxia, and } \\
\text { blurred vision }\end{array}$ & $\begin{array}{l}\text { Small dose } \\
\text { well tolerated }\end{array}$ \\
\hline
\end{tabular}


South American Journal of Clinical Research

Volume 3, Issue 1, 2016

Description of open label clinical studies

\begin{tabular}{|c|c|c|c|c|c|c|c|c|c|c|}
\hline S.No. & Study & $\begin{array}{l}\text { Indicati } \\
\text { on }\end{array}$ & $\begin{array}{l}\text { Populatio } \\
\text { n }\end{array}$ & $\begin{array}{l}\text { Study } \\
\text { Design }\end{array}$ & $\begin{array}{l}\text { Intervention } \\
+ \\
\text { Route of } \\
\text { Administrati } \\
\text { on }\end{array}$ & Intervention & Control & Results & $\begin{array}{l}\text { Adverse } \\
\text { Events }\end{array}$ & Significance \\
\hline 1. & $\begin{array}{l}\text { Waissengri } \\
\text { n, B. et al. } \\
2015 \\
{[40] \text {. }}\end{array}$ & $\begin{array}{l}\text { Advanc } \\
\text { ed } \\
\text { Cancer }\end{array}$ & $\begin{array}{l}113 \\
\text { patients } \\
\text { with } \\
\text { cancer }\end{array}$ & $\begin{array}{l}\text { Open- } \\
\text { label } \\
\text { study }\end{array}$ & $\begin{array}{l}\text { Cannabis } \\
\text { orally and by } \\
\text { inhalation }\end{array}$ & $\begin{array}{l}\text { Detailed } \\
\text { Questionnaire }\end{array}$ & $\mathrm{Nil}$ & $\begin{array}{l}\text { Pain } \\
\text { reduction, } \\
\text { Improvement } \\
\text { in appetite, } \\
\text { Reduced } \\
\text { nausea }\end{array}$ & $\begin{array}{l}\text { fatigue and } \\
\text { dizziness }\end{array}$ & $\begin{array}{l}\text { Highly } \\
\text { effective }\end{array}$ \\
\hline 2. & $\begin{array}{l}\text { Johnson, } \\
\text { J.R. et al. } \\
2013 \\
{[41] \text {. }}\end{array}$ & Cancer & $\begin{array}{l}43 \\
\text { patients } \\
\text { with } \\
\text { chronic } \\
\text { cancer } \\
\text { pain }\end{array}$ & $\begin{array}{l}\text { Open- } \\
\text { label } \\
\text { study }\end{array}$ & $\begin{array}{l}\text { Cannabis } \\
\text { Sublingually }\end{array}$ & $\begin{array}{l}\text { THC/CBD } \\
\text { spray }(n=39) \\
+ \\
\text { THC spray } \\
(n=4)\end{array}$ & Placebo & $\begin{array}{l}\text { Improvement } \\
\text { in insomnia, } \\
\text { pain, and } \\
\text { fatigue }\end{array}$ & $\begin{array}{l}\text { None serious } \\
\text { side effect }\end{array}$ & $\begin{array}{l}\text { Long-term } \\
\text { use Well } \\
\text { tolerated }\end{array}$ \\
\hline 3. & $\begin{array}{l}\text { Maida, V. } \\
\text { et al. } \\
2008 \\
{[42] .}\end{array}$ & Cancer & $\begin{array}{l}112 \\
\text { patients } \\
\text { with } \\
\text { advanced } \\
\text { cancer }\end{array}$ & $\begin{array}{l}\text { Open- } \\
\text { label } \\
\text { study }\end{array}$ & $\begin{array}{l}\text { Nabilone } \\
\text { orally }\end{array}$ & $\begin{array}{l}\text { Nabilone } \\
(\mathrm{n}=47)\end{array}$ & $\begin{array}{l}\text { Placebo } \\
(n=65)\end{array}$ & $\begin{array}{l}\text { Improvement } \\
\text { in appetite } \\
\text { and reduction } \\
\text { in pain, } \\
\text { nausea, } \\
\text { anxiety and } \\
\text { overall } \\
\text { distress }\end{array}$ & $\begin{array}{l}\text { no relevant } \\
\text { side-effects }\end{array}$ & $\begin{array}{l}\text { Significant } \\
\text { improvement } \\
\text { of pain }\end{array}$ \\
\hline 4. & $\begin{array}{l}\text { Maida, V. } \\
2008\end{array}$ & Cancer & $\begin{array}{l}4 \\
\text { advanced }\end{array}$ & $\begin{array}{l}\text { Open- } \\
\text { label }\end{array}$ & $\begin{array}{l}\text { Nabilone } \\
\text { orally }\end{array}$ & $\begin{array}{l}\text { synthetic orally } \\
\text { administered }\end{array}$ & Nil & $\begin{array}{l}\text { Significant } \\
\text { improvement }\end{array}$ & None & $\begin{array}{l}\text { Well } \\
\text { tolerated }\end{array}$ \\
\hline
\end{tabular}


South American Journal of Clinical Research

Volume 3, Issue 1, 2016

\begin{tabular}{|c|c|c|c|c|c|c|c|c|c|c|}
\hline & [43]. & & $\begin{array}{l}\text { cancer } \\
\text { patients } \\
\text { with } \\
\text { severe } \\
\text { night } \\
\text { sweats }\end{array}$ & study & & $\begin{array}{l}\text { cannabinoid } \\
\text { Nabilone } \\
(\mathrm{n}=4)\end{array}$ & & $\begin{array}{l}\text { of night } \\
\text { sweats within } \\
2 \text { days }\end{array}$ & & \\
\hline 5. & $\begin{array}{l}\text { Engels, } \\
\text { F.K. et al. } \\
2007 \\
{[44] .}\end{array}$ & Cancer & $\begin{array}{l}24 \text { cancer } \\
\text { patients } \\
\text { treated } \\
\text { with } \\
\text { irinotecan } \\
\text { or } \\
\text { docetaxel }\end{array}$ & $\begin{array}{l}\text { Open- } \\
\text { label } \\
\text { study }\end{array}$ & $\begin{array}{l}\text { Cannabis } \\
\text { orally as } \\
\text { herbal tea }\end{array}$ & $\begin{array}{l}\text { irinotecan }(600 \\
\text { mg, } \mathrm{n}=12)+ \\
\text { medicinal } \\
\text { cannabis; } \\
\text { docetaxel }(180 \\
\text { mg, } \mathrm{n}=12)+ \\
\text { medicinal } \\
\text { cannabis }\end{array}$ & Placebo & $\begin{array}{l}\text { Reduction in } \\
\text { pain }\end{array}$ & $\begin{array}{l}\text { no relevant } \\
\text { side-effects }\end{array}$ & $\begin{array}{l}\text { Well } \\
\text { tolerated }\end{array}$ \\
\hline 6. & $\begin{array}{l}\text { Maida V. } \\
2006 \\
{[45] .}\end{array}$ & Cancer & $\begin{array}{l}139 \\
\text { cancer } \\
\text { patients }\end{array}$ & $\begin{array}{l}\text { Open- } \\
\text { label } \\
\text { study }\end{array}$ & $\begin{array}{l}\text { Nabilone } \\
\text { orally }\end{array}$ & $\begin{array}{l}\text { Nabilone } \\
(\mathrm{n}=82)\end{array}$ & $\begin{array}{l}\text { Placebo } \\
(\mathrm{n}=57)\end{array}$ & $\begin{array}{l}\text { improved } \\
\text { pain, nausea, } \\
\text { insomnia, } \\
\text { night sweats, } \\
\text { distress } \\
\end{array}$ & $\begin{array}{l}\text { Anxiety and } \\
\text { Depression }\end{array}$ & $\begin{array}{l}\text { Well } \\
\text { tolerated }\end{array}$ \\
\hline 8. & $\begin{array}{l}\text { Guzman, } \\
\text { M. et al. } \\
2006 \\
{[47] \text {. }}\end{array}$ & Cancer & $\begin{array}{l}9 \text { patients } \\
\text { with } \\
\text { glioblasto } \\
\text { ma } \\
\text { multiform } \\
\text { e (brain }\end{array}$ & $\begin{array}{l}\text { Open- } \\
\text { label } \\
\text { study }\end{array}$ & $\begin{array}{l}\text { Delta-9-THC } \\
\text { intratumoraly }\end{array}$ & $\begin{array}{l}\text { Delta-9-THC } \\
\mathrm{n}=9\end{array}$ & $\begin{array}{l}\text { After } 24 \\
\text { weeks } \\
\text { (dose } \\
\text { escalation } \\
\text { regimen) }\end{array}$ & $\begin{array}{l}\text { antiproliferati } \\
\text { ve action on } \\
\text { tumor cells }\end{array}$ & $\begin{array}{l}\text { no relevant } \\
\text { side-effects }\end{array}$ & $\begin{array}{l}\text { Well } \\
\text { tolerated }+ \\
\text { safety profile }\end{array}$ \\
\hline
\end{tabular}


South American Journal of Clinical Research

Volume 3, Issue 1, 2016

\begin{tabular}{|c|c|c|c|c|c|c|c|c|c|c|}
\hline & & & tumor) & & & & & & & \\
\hline 9. & $\begin{array}{l}\text { Musty, } \\
\text { R.E.\& } \\
\text { Rossi, R. et } \\
\text { al. } \\
2001 \\
\text { [48]. }\end{array}$ & Cancer & $\begin{array}{l}1093 \\
\text { patients }\end{array}$ & $\begin{array}{l}\text { Open- } \\
\text { label } \\
\text { study }\end{array}$ & $\begin{array}{l}\text { Inhalation of } \\
\text { Cannabis+ } \\
\text { Delta-9-THC } \\
\text { Orally }\end{array}$ & $\begin{array}{l}\text { Inhalation of } \\
\text { Cannabis } \\
(\mathrm{n}=748) ; \\
\text { oral THC } \\
\text { capsule } \\
(\mathrm{n}=345)\end{array}$ & placebo & $\begin{array}{l}\text { relief from } \\
\text { nausea and } \\
\text { vomiting }\end{array}$ & $\begin{array}{l}\text { no relevant } \\
\text { side-effects }\end{array}$ & $\begin{array}{l}\text { Canbe used } \\
\text { as an add-on } \\
\text { therapy for } \\
\text { relief from } \\
\text { nausea and } \\
\text { vomiting }\end{array}$ \\
\hline 10. & $\begin{array}{l}\text { Abrahamov } \\
\text {, A. et al. } \\
1995 \\
{[49] .}\end{array}$ & Cancer & $\begin{array}{l}8 \text { children } \\
\text { with } \\
\text { cancer }\end{array}$ & $\begin{array}{l}\text { Open- } \\
\text { label } \\
\text { study }\end{array}$ & $\begin{array}{l}\text { Delta-9-THC } \\
\text { Orally }\end{array}$ & $\begin{array}{l}\text { Delta-9-THC } \\
2 \text { hours before } \\
\text { chemotherapy }\end{array}$ & $\begin{array}{l}\text { From } \\
\text { baseline }\end{array}$ & $\begin{array}{l}\text { complete } \\
\text { prevention of } \\
\text { vomiting }\end{array}$ & $\begin{array}{l}\text { no relevant } \\
\text { side-effects }\end{array}$ & $\begin{array}{l}\text { Well } \\
\text { tolerated }\end{array}$ \\
\hline 11. & $\begin{array}{l}\text { Nelson, K. } \\
\text { et al. } \\
1994 \\
{[50] .}\end{array}$ & Cancer & $\begin{array}{l}18 \\
\text { patients } \\
\text { with } \\
\text { cancer } \\
\end{array}$ & $\begin{array}{l}\text { Open- } \\
\text { label } \\
\text { study }\end{array}$ & $\begin{array}{l}\text { Delta-9-THC } \\
\text { Orally }\end{array}$ & Delta-9-THC & $\begin{array}{l}\text { one hour } \\
\text { after meals } \\
\text { for four } \\
\text { weeks }\end{array}$ & $\begin{array}{l}\text { Increased } \\
\text { appetite }\end{array}$ & $\begin{array}{l}\text { no relevant } \\
\text { side-effects }\end{array}$ & $\begin{array}{l}\text { effective } \\
\text { appetite } \\
\text { stimulant }\end{array}$ \\
\hline 12. & $\begin{array}{l}\text { Wadleigh, } \\
\text { R. et al. } \\
1990 \\
{[51] \text {. }}\end{array}$ & Cancer & $\begin{array}{l}30 \text { cancer } \\
\text { patients. }\end{array}$ & $\begin{array}{l}\text { Open- } \\
\text { label } \\
\text { study }\end{array}$ & $\begin{array}{l}\text { Delta-9-THC } \\
\text { Orally }\end{array}$ & dronabinol & placebo & $\begin{array}{l}\text { stimulated } \\
\text { mood and } \\
\text { appetite }\end{array}$ & Weight loss & $\begin{array}{l}\text { Well } \\
\text { tolerated }\end{array}$ \\
\hline 13. & $\begin{array}{l}\text { Cunningha } \\
\text { m, D. et al. } \\
1988 \\
{[52] \text {. }}\end{array}$ & Cancer & $\begin{array}{l}80 \\
\text { chemother } \\
\text { apy } \\
\text { patients }\end{array}$ & $\begin{array}{l}\text { Open- } \\
\text { label } \\
\text { study }\end{array}$ & $\begin{array}{l}\text { Nabilone } \\
\text { orally }\end{array}$ & $\begin{array}{l}\text { Nabilone }+ \\
\text { prochlorperazin } \\
\text { e }\end{array}$ & $\begin{array}{l}\text { Placebo } \\
\text { (metoclopr } \\
\text { amide and } \\
\text { dexamethas } \\
\text { one) }\end{array}$ & $\begin{array}{l}\text { Complete } \\
\text { control of } \\
\text { nausea and } \\
\text { vomiting }\end{array}$ & $\begin{array}{l}\text { no relevant } \\
\text { side-effects }\end{array}$ & $\begin{array}{l}\text { Better } \\
\text { tolerated }\end{array}$ \\
\hline 14. & $\begin{array}{l}\text { Vinciguerra } \\
\text {, V.et al. } \\
1988 \\
{[53] \text {. }}\end{array}$ & Cancer & $\begin{array}{l}56 \\
\text { patients } \\
\text { with } \\
\text { cancer } \\
\end{array}$ & $\begin{array}{l}\text { Open- } \\
\text { label } \\
\text { study }\end{array}$ & $\begin{array}{l}\text { Inhalation of } \\
\text { Cannabis }\end{array}$ & $\begin{array}{l}\text { Inhaled } \\
\text { Cannabis }\end{array}$ & Placebo & $\begin{array}{l}\text { Reduction in } \\
\text { nausea and } \\
\text { vomiting }\end{array}$ & $\begin{array}{l}\text { Sedation and } \\
\text { xerostomia }\end{array}$ & $\begin{array}{l}\text { Well } \\
\text { tolerated }\end{array}$ \\
\hline 15. & $\begin{array}{l}\text { Priestman, } \\
\text { T.J., \& }\end{array}$ & Cancer & $\begin{array}{l}30 \\
\text { patients }\end{array}$ & $\begin{array}{l}\text { Open- } \\
\text { label }\end{array}$ & $\begin{array}{l}\text { Nabilone } \\
\text { orally }\end{array}$ & Nabilone & $\begin{array}{l}\text { Placebo } \\
\text { (metoclopr }\end{array}$ & $\begin{array}{l}\text { Reduction in } \\
\text { resistant }\end{array}$ & $\begin{array}{l}\text { Minimal side } \\
\text { effects }\end{array}$ & $\begin{array}{l}\text { Efficacy of } \\
\text { Nabilone }\end{array}$ \\
\hline
\end{tabular}


South American Journal of Clinical Research

Volume 3, Issue 1, 2016

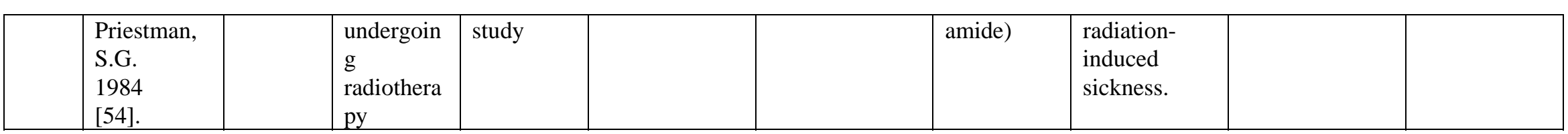

\section{Description of uncontrolled case reports:}

\begin{tabular}{|c|c|c|c|c|c|c|c|c|c|c|}
\hline S.No. & Study & $\begin{array}{l}\text { Indicatio } \\
\text { n }\end{array}$ & $\begin{array}{l}\text { Populatio } \\
\text { n }\end{array}$ & $\begin{array}{l}\text { Study } \\
\text { Design }\end{array}$ & $\begin{array}{l}\text { Intervention } \\
+ \\
\text { Route of } \\
\text { Administrati } \\
\text { on }\end{array}$ & $\begin{array}{l}\text { Interventio } \\
\mathbf{n}\end{array}$ & Control & Results & $\begin{array}{l}\text { Adverse } \\
\text { Events }\end{array}$ & Significance \\
\hline 1. & $\begin{array}{l}\text { Gottschling } \\
\text {, S. } \\
2011 \\
\text { [55]. }\end{array}$ & Cancer & $\begin{array}{l}50 \\
\text { children } \\
\text { with } \\
\text { cancer }\end{array}$ & $\begin{array}{l}\text { Uncontrolled } \\
\text { case report }\end{array}$ & $\begin{array}{l}\text { Delta-9-THC } \\
\text { Orally }\end{array}$ & $\begin{array}{l}\text { Dronabinol } \\
\text { dose was } 0.2 \\
\text { mg/kg } \\
\text { bodyweight } \\
\text { in children }\end{array}$ & Placebo & $\begin{array}{l}\text { Reduced } \\
\text { pain, } \\
\text { spasticity and } \\
\text { improved } \\
\text { appetite and } \\
\text { nausea }\end{array}$ & $\begin{array}{l}\text { no relevant } \\
\text { side-effects }\end{array}$ & $\begin{array}{l}\text { No relevant } \\
\text { side effects } \\
\text { on long-term } \\
\text { treatment }\end{array}$ \\
\hline 2. & $\begin{array}{l}\text { Gonzalez- } \\
\text { Rosales, F., } \\
\text { \& Walsh, } \\
\text { D. } \\
1997 \\
{[56] .}\end{array}$ & Cancer & $\begin{array}{l}1 \text { patient } \\
\text { (whole } \\
\text { brain } \\
\text { radiation) }\end{array}$ & $\begin{array}{l}\text { Uncontrolled } \\
\text { case report }\end{array}$ & $\begin{array}{l}\text { Delta-9-THC } \\
\text { Orally }\end{array}$ & Dronabinol & Placebo & $\begin{array}{l}\text { Relief from } \\
\text { nausea and } \\
\text { vomiting }\end{array}$ & $\begin{array}{l}\text { no relevant } \\
\text { side-effects }\end{array}$ & $\begin{array}{l}\text { Well } \\
\text { tolerated }\end{array}$ \\
\hline
\end{tabular}


South American Journal of Clinical Research

Volume 3, Issue 1, 2016

\section{Data interpretation}

On the basis of the data collected and retrieved, following observations were made in the form of plots and graphs.

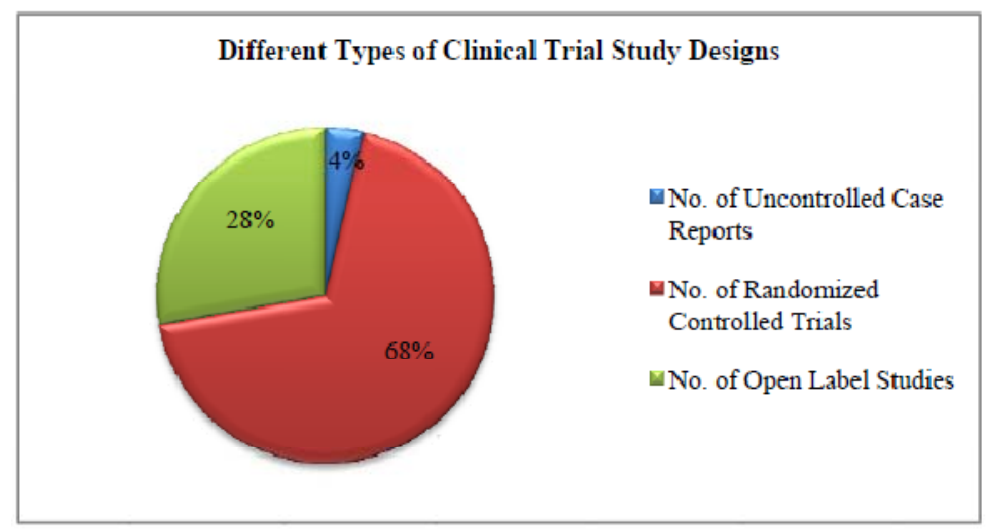

Fig. 2. Different Clinical Study Designs[3-56].

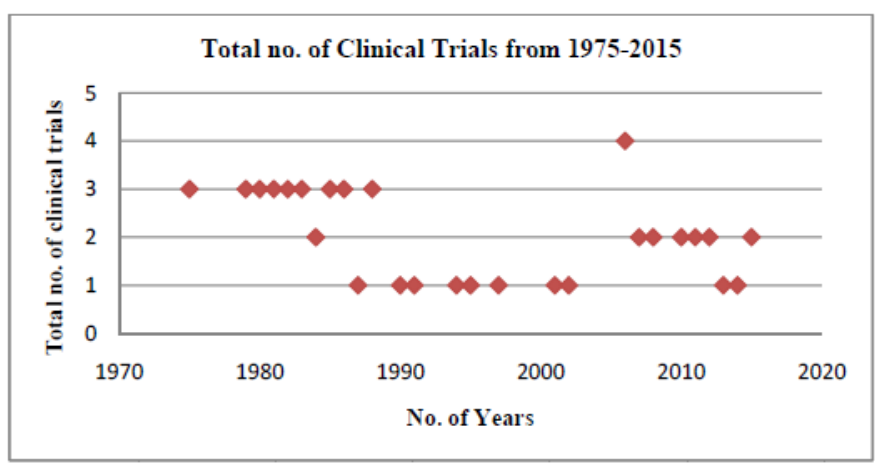

Fig. 3. Clinical Trials from 1975-2015[3-56].

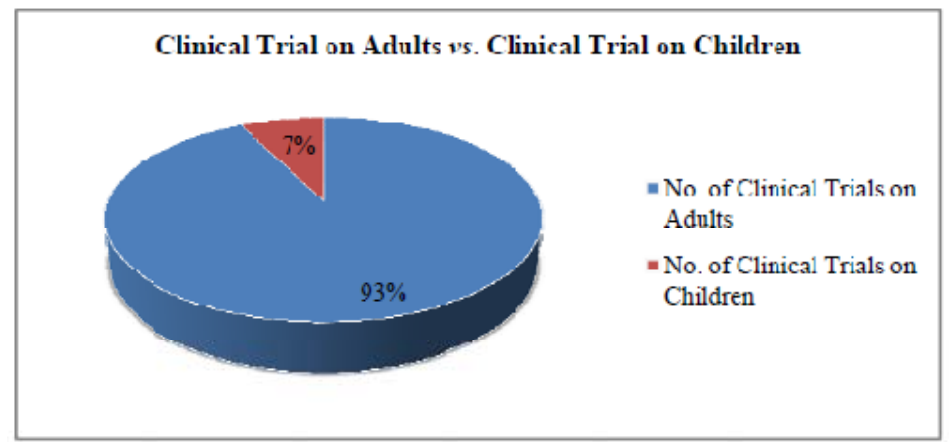

Fig. 4. Adults Clinical Trials vs. Chidren Clinical Trials[3-56]. 


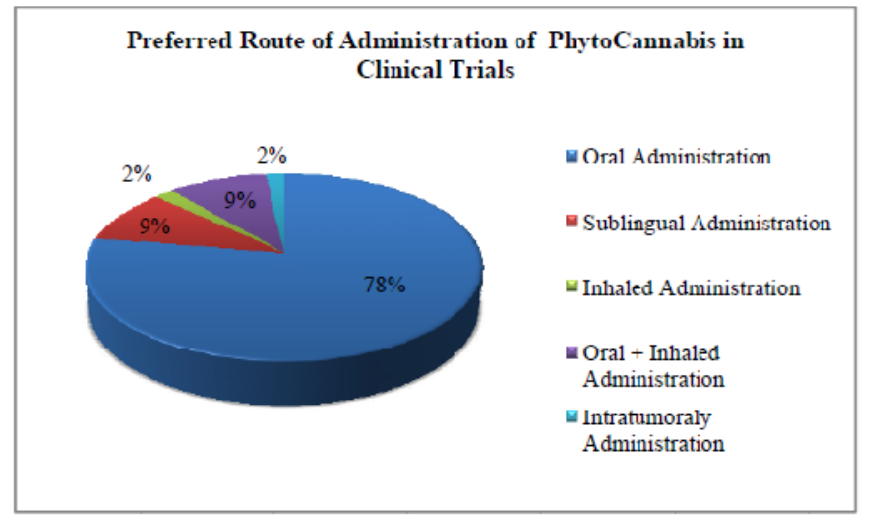

Fig. 5 Preferred Route of Administration of PhytoCannabis[3-56].

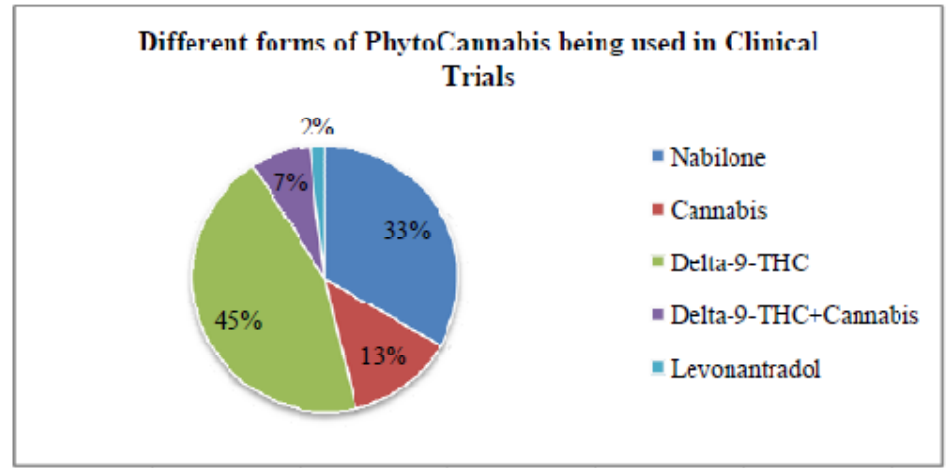

Fig. 6. Different forms of PhytoCannabis Used[3-56].

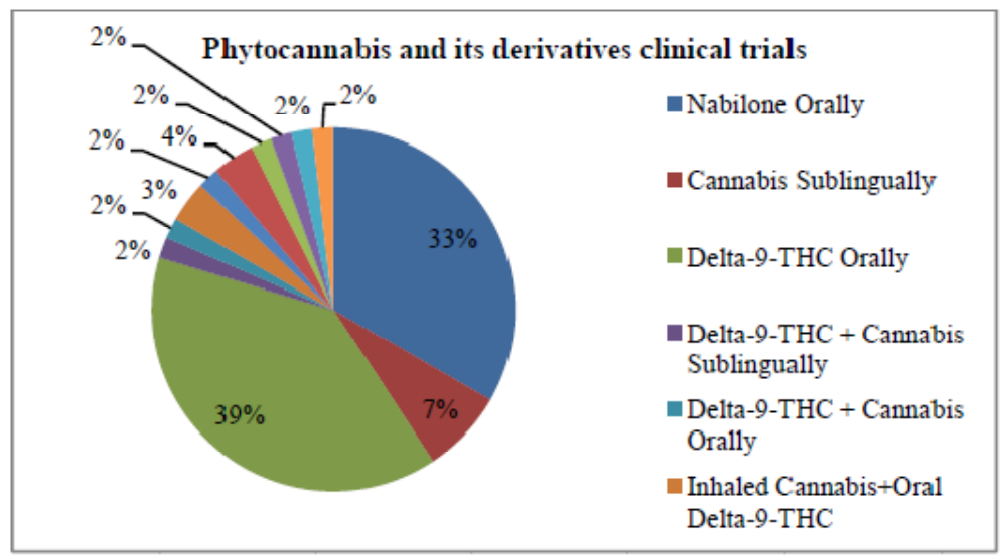

Fig. 7. Phytocannabis and its derivatives clinical trials[3-56]. 
South American Journal of Clinical Research

Volume 3, Issue 1, 2016

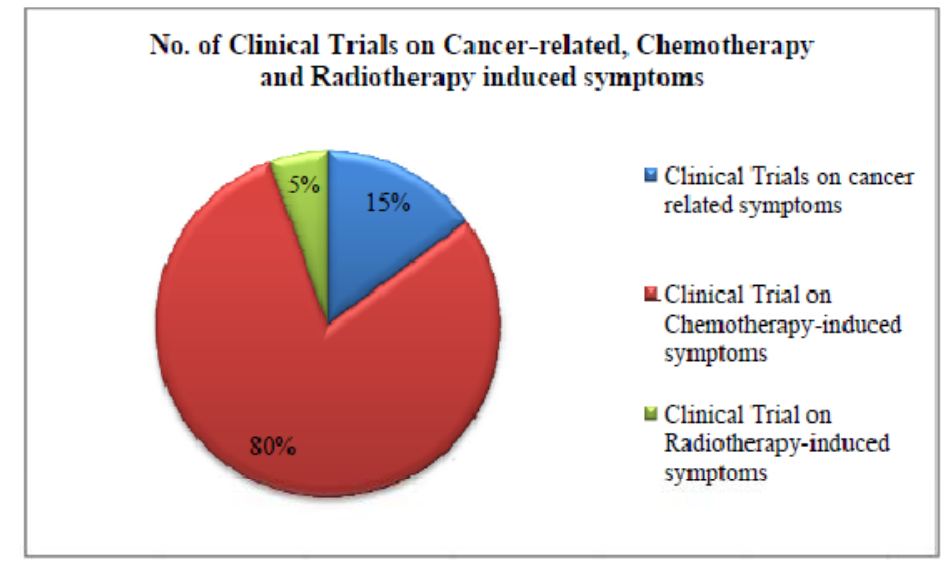

Fig. 8 No. of Clinical Trials on Cancer-related, Chemotherapy and Radiotherapy induced symptoms[356].

\section{Discussion}

From the literature and data reviewed, it was observed that 37 studies were identified with Phytocannabis use in cancer treatment, amongst which only 2 trials reported for its efficacy in children as compared to that on adults in 35 trials [3-39]. Nabilone (Phytocannabis) when administered orally was well tolerated with marked improvement in pain intensity, improved appetite, reduced nausea and vomiting along with minimal side effects such as, dizziness, drowsiness, hallucinations, dry mouth, vertigo, postural hypotension, sleep disturbances and fatigue[3-39]. In some studies, where no significant improvement was observed, still the patient's choice of preference was Oral Nabilone in either capsule form or as herbal tea [339]. When Phytocannabis (Cannabis) was administered sublingually in the form of Sprays, there was marked great reduction in pain intensity accompanied by reduced nausea and vomiting along with minimal side effects of loss in appetite [3-39]. When Phytocannabis (Delta-9-THC) was administered orally was found to be useful in palliation of chemosensory alterations and improved food taste and appetite, reduced nausea and vomiting along with less concentration ability, less social interaction, sedation, mental clouding and blurred vision [339]. In a study conducted by Johnson, J.R. et al. (2010), THC: CBD was found to be more effective in reduction of pain in cancer patients [8]. On the other hand, mild psychological effects were found to be associated with inhaled form of Phytocannabis [8].

On the other hand, it was observed that 2 uncontrolled case reports presented with intervention of Phytocannabis (Delta-9-THC) led to reduction in nausea and vomiting that was induced by chemotherapy and radiotherapy treatments [40-54]. It was also found that due to this intervention there was marked improvement in appetite and pain [40-54]. From the open-labeled studies conducted, it was observed that 15 studies were identified from the year 1984to 2015 for the use of Phytocannabis in the treatment of cancer and treatment related side effects [40-54]. Moreover, it was observed that Nabilone when administered orally was well tolerated with significant improvement in pain intensity, reduced nausea and vomiting, improved insomnia and night sweats, reduced distress and reduced radiotherapy-induced sickness[40-54]. The intervention of the Phytocannabis Nabilone orally produced mild side effects of anxiety and depression [40-54]. Secondly, when Phytocannabis was administered orally, inhaled/smoked or sublingually, it was highly effective in reduction of pain intensity along with improved appetite, reduced nausea and vomiting, with minimal side effects such as sedation, dizziness and Xerostomia [40-54]. Another form of Phytocannabis, namely, Delta9-THC when administered orally was also well tolerated by increased appetite and reduced nausea and vomiting with minor side effects of weight loss and dizziness [40-54]. According to Guzman, M. et al. (2006)study, Phytocannabis in the form of Delta-9-THC when administered intracranially to the brain tumor patients after undergoing Whole Brain 
Radiotherapy showed anti-proliferative action on tumor cells thereby reduced the associated symptoms of Radiotherapy [18]. However, much insight into its effectiveness is required for its standardization [18].

Moreover, it was seen that only two out of 15 clinical trials were conducted on children suffering from cancer $[55,56]$. This in part reflected difficulties in collecting comparable data onillicit drug usage [55, 56, 57]. Some countries did not conduct surveys of drug use, some conducted surveys annually and others conducted them less frequently [55, 56, 57]. Of those surveys that were conducted, there was variation between countries in assessing frequency of use, and age groups were divided differently or differed in the settings in which the adolescents and young adults were surveyed [55, 56, 57].

On the whole, it could be concluded from this presented report that there had been more number of Randomized controlled trials in comparison to open-labelled and uncontrolled case studies as retrieved from 1975-2015. During those subsequent 40 years, there were comparatively less number of clinical trials for the use of Phytocannabis and its derivatives for the cancer-related and cancer treatment related studies as compared to the increased burden of the disease globally. Moreover, only few studies focused on the subjects that consisted children which was a major pitfall in finding the compassionate treatment for children group as well who were at the same risk level of developing the disease. Phytocannabis (Nabilone) oral route of administration either alone or as add-on therapy was found to be more preferred, safe and effective to be used in patients who underwent either chemotherapy or radiotherapy treatment.

\section{Conclusion}

The presented report contributed to the development of evidence based use of Phytocannabis for cancer treatment which ultimately contributed to the improvement of the quality of life of people suffering from cancer who underwent either chemotherapy or radiotherapy treatments. Therefore, it was concluded that the treatment decisions should be based on standard principles of medical-care ethics - that provided equitable access to treatment and psychosocial support that best meet the needs of the individual cancer patient [58]. Treatment should respect and validate the autonomy of the individual, with patients being fully informed about the risks and benefits of treatment choices [58]. The development and maintenance of Phytocannabis treatment services evidently needs to take place within the broader system of health-care financing and provision in a given country [58]. The presented report thus, highlighted the benefic use of Phytocannabis and its derivatives in the treatment of cancer patients thereby by enhanced quality of life of those patients with minimal side effects being associated with the prolonged disease. In a nutshell, Phytocannabis (Nabilone, Delta-9-THC, Cannabis) in the form of capsules or inhaled form was found to be safe and effective to be used in Metastatic cancer, Advanced cancer, Neuropathic pain, nausea and vomiting induced by Chemotherapy and radiotherapy, Head and Neck cancer, Malignant Melanoma, Hematologic cancer, Brain tumor, Gastrointestinal carcinoma, Osteogenic sarcoma, Liver cancer, Bone cancer, Lungs cancer, Non-seminatous Testicular cancer, Abdominal cancer and Soft tissue sarcoma.

\section{Future directions}

There were certain areas that required more research as mentioned below:

1. There were none to minimal standard measures of the Phytocannabis and its content used in most of the countries and regions worldwide.

2. Global data were required on the frequency of Phytocannabis use (more than once daily, daily, near daily, weekly, etc.) and the prevalence of health and social consequences.

3. Data was required on the typical doses of Phytocannabis (smoked, vaporized, ingested) with the potency of Phytocannabis for long term usage and its impact on 
South American Journal of Clinical Research

Volume 3, Issue 1, 2016

health and quality of life of the cancer patients. Larger cohort and better designed case-control studies were needed to better understand the control for benefic effects.

4. Global assessments were needed to establish the relationship between the use of Phytocannabis and other conventional drugs.

5. Most of the studies on risk and protective factors for Phytocannabis use had been conducted in a limited number of high-income countries. There was some uncertainty as to whether the same risk factors prevailed in low- and middle-income countries. More research was required on Phytocannabis use in low- and middle-income countries for the treatment of cancer and related symptoms.

\section{References}

[1] Abrahamov, A., Mechoulam, R. (1995). An efficient new cannabinoid antiemetic in pediatric oncology. Life Sciences, 56(23-24):2097-2102. Available from: http://www.cannabismed.org/studies/ww_en_db_study_show.php?s_id=7 .

[2] Ahmedzai, S., Carlyle, D.L., Calder, I.T., Moran, F. (1983). Anti-emetic efficacy and toxicity of nabilone, a synthetic cannabinoid, in lung cancer chemotherapy. Br J Cancer, 48(5):657-63. Available from: http://www.cannabis-med.org/studies/ww_en_db_study_show.php?s_id=119 .

[3] Brisbois, T.D., de Kock, I.H., Watanabe, S.M., Mirhosseini, M., Lamoureux, D.C., Chasen, M., Macdonald, N., Baracos, V.E., Wismer, W.V. (2011). Delta-9-tetrahydrocannabinol may palliate altered chemosensory perception in cancer patients: results of a randomized, double-blind, placebocontrolled pilot trial. Ann Oncol., 22(9):2086-93. Available from: http://www.cannabismed.org/studies/ww_en_db_study_show.php?s_id=304

[4] Cancer (Fact Sheets). Updated February 2015. Accessed on: 2016 June 1. Available from: http://www.who.int/mediacentre/factsheets/fs297/en/ .

[5] Chang, A.E., Shiling, D.J., Stillman, R.C., Goldberg, N.H., Seipp, C.A., Barofsky, I., Rosenberg. (1981). A prospective evaluation of delta-9-tetrahydrocannabinol as an antiemetic in patients receiving adriamycin and cytoxan chemotherapy. Cancer, 47(7):1746-51. Available from: http://www.cannabismed.org/studies/ww_en_db_study_show.php?s_id=22 .

[6] Chang, A.E., Shiling, D.J., Stillman, R.C., Goldberg, N.H., Seipp, C.A., Barofsky, I., Simon, R.M., Rosenberg, S.A. (1979). Delta-9-tetrahydrocannabinol as an antiemetic in cancer patients receiving high-dose methotrexate. A prospective, randomized evaluation. Annals of Internal Medicine, 91:819824. Available from: http://www.cannabis-med.org/studies/ww_en_db_study_show.php?s_id=23 .

[7] Chan, H.S., Correia, J.A., MacLeod, S.M. (1987). Nabilone versus prochlorperazine for control of cancer chemotherapy-induced emesis in children: a double-blind, crossover trial. Pediatrics, 79(6):94652. Available from: http://www.cannabis-med.org/studies/ww_en_db_study_show.php?s_id=120 .

[8] Citron, M.L., Herman, T.S., Vreeland, F., Krasnow, S.H., Fossieck, B.E. Jr, Harwood, S., Franklin, R., Cohen, M.H. (1985). Antiemetic efficacy of levonantradol compared to delta-9tetrahydrocannabinol for chemotherapy-induced nausea and vomiting. Cancer Treat Rep., 69(1):10912. Available from: http://www.cannabis-med.org/studies/ww_en_db_study_show.php?s_id=152.

[9] Côté, M., Trudel, M., Wang, C., Fortin, A. (2015). Improving Quality of Life With Nabilone During Radiotherapy Treatments for Head and Neck Cancers: A Randomized Double-Blind PlaceboControlled Trial. Ann Otol Rhinol Laryngol, 125(4):317-24. doi: http://10.1177/0003489415612801. [ PubMed PMID: 26503964].

[10] Cunningham, D., Bradley, C.J., Forrest, G.J., Hutcheon, A.W., Adams, L., Sneddon, M., Harding, M., Kerr, D.J., Soukop, M., Kaye, S.B. (1988). A randomized trial of oral nabilone and prochlorperazine compared to intravenous metoclopramide and dexamethasone in the treatment of nausea and vomiting induced by chemotherapy regimens containing cisplatin or cisplatin analogues.

Eur J Cancer Clin Oncol., 24(4):685-9. Available from: http://www.cannabismed.org/studies/ww_en_db_study_show.php?s_id=122 .

[11] Dalzell, A.M., Bartlett, H., Lilleyman, J.S. (1986). Nabilone: an alternative antiemetic for cancer chemotherapy. Arch Dis Child, 61(5):502-5. Available from:

http://www.cannabis-med.org/studies/ww_en_db_study_show.php?s_id=123 . 
[12] Duran, M., Pérez, E., Abanades, S., Vidal, X., Saura, C., Majem, M., Arriola, E., Rabanal, M., Pastor, A., Farré, M., Rams, N., Laporte, J.R., Capellà, D. (2010). Preliminary efficacy and safety of an oromucosal standardized cannabis extract in chemotherapy-induced nausea and vomiting. $\mathrm{Br} J \mathrm{Clin}$ Pharmacol., 70(5):656-63. Available from:

http://www.cannabis-med.org/studies/ww_en_db_study_show.php?s_id=312 .

[13] Einhorn, L.H., Nagy, C., Furnas, B., Williams, S.D. (1981). Nabilone: an effective antiemetic in patients receiving cancer chemotherapy. J Clin Pharmacol, 21(8-9 Suppl):64S-69S. Available from: http://www.cannabis-med.org/studies/ww_en_db_study_show.php?s_id=124 .

[14] Engels, F.K., de Jong, F.A., Sparreboom, A., Mathot, R.A., Loos, W.J., Kitzen, J.J., de Bruijn, P., Verweij, J., Mathijssen, R.H. (2007). Medicinal cannabis does not influence the clinical pharmacokinetics of irinotecan and docetaxel. Oncologist, 12(3):291-300. Available from: http://www.cannabis-med.org/studies/ww_en_db_study_show.php?s_id=246 .

[15] Epidemiology of cannabis use, disorders and treatment. Updated February 2015. Accessed on: 2016 June 1. Available from:

http://www.who.int/substance_abuse/publications/cannabis_report/en/index5.html .

[16] Frytak, S., Moertel, C.G., O'Fallon, J.R., Rubin, J., Creagan, E.T., O'Connell, M.J., Schutt, A.J., Schwartau, N.W. (1979). Delta-9-tetrahydrocannabinol as an antiemetic for patients receiving cancer chemotherapy. A comparison with prochlorperazine and a placebo. Annals of Internal Medicine, 91(6):825-830. Available from:

http://www.cannabis-med.org/studies/ww_en_db_study_show.php?s_id=5 .

[17] George, M., Pejovic, M.H., Thuaire, M., Kramar, A., Wolff, J.P. (1983). Randomized comparative trial of a new anti-emetic: nabilone, in cancer patients treated with cisplatin. Biomed Pharmacother, 37(1):24-7. Available from:

http://www.cannabis-med.org/studies/ww_en_db_study_show.php?s_id=125 .

[18] Gonzalez-Rosales, F., Walsh, D. (1997). Intractable nausea and vomiting due to gastrointestinal mucosal metastases relieved by tetrahydrocannabinol (dronabinol). Journal of Pain and Symptom Management, 14(5):311-314. Available from:

http://www.cannabis-med.org/studies/ww_en_db_study_show.php?s_id=35 .

[19] Gottschling, S. (2011). Cannabinoids in children. Applied pain management and palliative care, 1:55-57. Available from: http://www.cannabis-med.org/studies/ww_en_db_study_show.php?s_id=295. [20] Guidelines for the Psychosocially Assisted Pharmacological Treatment of Opioid Dependence. (2009). Available from:

http://www.who.int/substance_abuse/publications/opioid_dependence_guidelines.pdf?ua=1 .

[21] Guzman, M., Duarte, M.J., Blazquez, C., Ravina, J., Rosa, M.C., Galve-Roperh, I., Sanchez, C., Velasco, G., Gonzalez-Feria, L. (2006). A pilot clinical study of Delta(9)-tetrahydrocannabinol in patients with recurrent glioblastoma multiforme. Br J Cancer, 95(2):197-203. Available from: http://www.cannabis-med.org/studies/ww_en_db_study_show.php?s_id=193 .

[22] Herman, T.S., Einhorn, L.H., Jones, S.E., Nagy, C., Chester, A.B., Dean, J.C., Furnas, B., Williams, S.D., Leigh, S.A., Dorr, R.T., Moon, T. E. (1979). Superiority of nabilone over prochlorperazine as an antiemetic in patients receiving cancer chemotherapy. $N$ Engl J Med., 300(23):1295-7. Available from:

http://www.cannabis-med.org/studies/ww_en_db_study_show.php?s_id=126 .

[23] Hutcheon, A.W., Palmer, J.B., Soukop, M., Cunningham, D., McArdle, C., Welsh, J., Stuart, F., Sangster, G., Kaye, S., Charlton, D. (1983). A randomised multicentre single blind comparison of a cannabinoid anti-emetic (levonantradol) with chlorpromazine in patients receiving their first cytotoxic chemotherapy. European Journal for Cancer and Clinical Oncology, 19(8):1087-90. Available from: http://www.cannabis-med.org/studies/ww_en_db_study_show.php?s_id=132 .

[24] Jatoi, A., Windschitl, H.E., Loprinzi, C.L., Sloan, J.A., Dakhil, S.R., Mailliard, J.A., Pundaleeka, S., Kardinal, C.G., Fitch, T.R., Krook, J.E., Novotny, P.J., Christensen, B. (2002). Dronabinol versus megestrol acetate versus combination therapy for cancer-associated anorexia: a North Central Cancer Treatment Group study. Journal of Clinical Oncology, 20(2):567-573. Available from: http://www.cannabis-med.org/studies/ww_en_db_study_show.php?s_id=49 . 
South American Journal of Clinical Research

Volume 3, Issue 1, 2016

[25] Johansson, R., Kilkku, P., Groenroos, M. (1982). A double-blind, controlled trial of nabilone vs. prochlorperazine for refractory emesis induced by cancer chemotherapy. Cancer Treat Rev., 9 Suppl B: 25-33. Available from: http://www.cannabis-med.org/studies/ww_en_db_study_show.php?s_id=146 .

[26] Johnson, J.R., Burnell-Nugent, M., Lossignol, D., Ganae-Motan, E.D., Potts, R., Fallon, M.T. (2010). Multicenter, Double-Blind, Randomized, Placebo-Controlled, Parallel-Group Study of the Efficacy, Safety, and Tolerability of THC: CBD Extract and THC Extract in Patients With Intractable Cancer-Related Pain. J Pain Symptom Manage, 39(2):167-79. Available from: http://www.cannabismed.org/studies/ww_en_db_study_show.php?s_id=368 .

[27] Johnson, J.R., Lossignol, D., Burnell-Nugent, M., Fallon, M.T. (2013). An open-label extension study to investigate the long-term safety and tolerability of THC/CBD oromucosal spray and oromucosal THC spray in patients with terminal cancer-related pain refractory to strong opioid analgesics. J Pain Symptom Manage, 46(2):207-18. doi: http://10.1016/j. [PubMed PMID: 23141881].

[28] Jones, S.E., Durant, J.R., Greco, F.A., Robertone, A. (1982). A multi-institutional Phase III study of nabilone vs. placebo in chemotherapy-induced nausea and vomiting. Cancer Treat Rev., 9 Suppl B: 45-8. Available from: http://www.cannabis-med.org/studies/ww_en_db_study_show.php?s_id=156 .

[29] Lane, M., Vogel, C.L., Ferguson, J., Krasnow, S., Saiers, J.L., Hamm, J. (1991). Dronabinol and prochlorperazine in combination for treatment of cancer chemotherapy-induced nausea and vomiting. Journal of Pain and Symptom Management,6:352-359. Available from: http://www.cannabismed.org/studies/ww_en_db_study_show.php?s_id=28 .

[30] Levitt, M., Faiman, C., Hawks, R., Wilson, A. (1984). Randomized double blind comparison of delta-9-tetrahydroicannabinol (THC) and marijuana as chemotherapy antiemetics. Proceedings of the American Society for Clinical Oncology,3:91. Available from: http://www.cannabismed.org/studies/ww_en_db_study_show.php?s_id=29 .

[31] Lynch, M.E., Cesar-Rittenberg, P., Hohmann, A.G. (2014). A double-blind, placebo-controlled, crossover pilot trial with extension using an oral mucosal cannabinoid extract for treatment of chemotherapy-induced neuropathic pain. J Pain Symptom Manage, 47(1):166-73. doi: http://10.1016/j. [PubMed PMID: 23742737].

[32] Madras, B.K. (2015). Update of Cannabis and its medical use. 37th ECDD (2015) Agenda item 6.2, available from:

http://www.who.int/medicines/access/controlled-substances/6_2_cannabis_update.pdf?ua=1\&ua=1 .

[33] Maida, V., Ennis, M., Irani, S., Corbo, M., Dolzhykov, M. (2008). Adjunctive nabilone in cancer pain and symptom management: a prospective observational study using propensity scoring. $J$ Support Oncol., 6(3):119-24. Available from:

http://www.cannabis-med.org/studies/ww_en_db_study_show.php?s_id=176 .

[34] Maida, V. (2008). Nabilone for the treatment of paraneoplastic night sweats: a report of four cases. J Palliat Med., 11(6):929-34. Available from:

http://www.cannabis-med.org/studies/ww_en_db_study_show.php?s_id=282 .

[35] Maida, V. (2006). The synthetic cannabinoid nabilone improves pain and symptom management in cancer patients. Abstract presented at the San Antonio Breast Cancer Symposium on 15 December 2006, Available from: http://www.cannabis-med.org/studies/ww_en_db_study_show.php?s_id=177 .

[36] McCabe, M., Smith, F.P., Goldberg, D., Macdonald, J., Woolley, P.V., Warren, R. (1988). Efficacy of tetrahydrocannabinol in patients refractory to standard anti-emetic therapy. Investigational New Drugs, 6:243-246. Available from:

http://www.cannabis-med.org/studies/ww_en_db_study_show.php?s_id=31 .

[37] Meiri, E., Jhangiani, H., Vredenburgh, J.J., Barbato, L.M., Carter, F.J., Yang, H.M., Baranowski, V. (2007). Efficacy of dronabinol alone and in combination with ondansetron versus ondansetron alone for delayed chemotherapy-induced nausea and vomiting. Curr Med Res Opin, 23(3):533-43. Available from: http://www.cannabis-med.org/studies/ww_en_db_study_show.php?s_id=191 .

[38] Musty, R.E., Rossi, R. (2001). Effects of smoked cannabis and oral delta-9-tetrahydrocannabinol on nausea and emesis after cancer chemotherapy: A review of state clinical trials. $J$ Cannabis Ther, 1(1):29-42. Available from:

http://www.cannabis-med.org/studies/ww_en_db_study_show.php?s_id=256 . 
[39] Neidhart, J.A., Gagen, M.M., Wilson, H.E., Young, D.C. (1981). Comparative trial of the antiemetic effects of THC and haloperidol. International Journal of Clinical Pharmacology Research, 21: 38-42S. Available from:

http://www.cannabis-med.org/studies/ww_en_db_study_show.php?s_id=64 .

[40] Nelson, K., Walsh, D., Deeter, P., Sheehan, F. (1994). A phase II study of delta-9tetrahydrocannabinol for appetite stimulation in cancer-associated anorexia. Journal of Palliative Care, 10(1):14-18. Available from:

http://www.cannabis-med.org/studies/ww_en_db_study_show.php?s_id=52 .

[41] Niederle, N., Schutte, J., Schmidt, C.G. (1986). Crossover comparison of the antiemetic efficacy of nabilone and alizapride in patients with nonseminomatous testicular cancer receiving cisplatin therapy. Klin Wochenschr, 64(8):362-5. Available from:

http://www.cannabis-med.org/studies/ww_en_db_study_show.php?s_id=127 .

[42] Niiranen, A., Mattson, K. (1985). A cross-over comparison of nabilone and prochlorperazine for emesis induced by cancer chemotherapy. Amerícan Journal of Clinical Oncology, 8(4):336-40. Available from: http://www.cannabis-med.org/studies/ww_en_db_study_show.php?s_id=128 .

[43] Noyes, R. Jr, Brunk, S.F., Avery, D.A.H., Canter, A. C. (1975). The analgesic properties of delta9-tetrahydrocannabinol and codeine. Clinical Pharmacology and Therapeutics, 18(1):84-89. Available from: http://www.cannabis-med.org/studies/ww_en_db_study_show.php?s_id=17 .

[44] Noyes, R. Jr, Brunk, S.F., Baram, D.A., Canter, A. (1975). Analgesic effect of delta-9tetrahydrocannabinol. Journal of Clinical Pharmacology, 15(2-3):139-143. Available from: http://www.cannabis-med.org/studies/ww_en_db_study_show.php?s_id=16 .

[45] Orr, L.E., McKernan, J.F., Bloome, B. (1980). Antiemetic effect of tetrahydrocannabinol. Compared with placebo and prochlorperazine in chemotherapy-associated nausea and emesis. Annals of Internal Medicine, 140(11):1431-1433. Available from:

http://www.cannabis-med.org/studies/ww_en_db_study_show.php?s_id=6 .

[46] Pomeroy, M., Fennelly, J.J., Towers, M. (1986). Prospective randomized double-blind trial of nabilone versus domperidone in the treatment of cytotoxic-induced emesis. Cancer Chemother Pharmacol, 17(3):285-8. Available from:

http://www.cannabis-med.org/studies/ww_en_db_study_show.php?s_id=129 .

[47] Portenoy, R.K., Ganae-Motan, E.D., Allende, S., Yanagihara, R., Shaiova, L., Weinstein, S., McQuade, R., Wright, S., Fallon, M.T. (2012). Nabiximols for opioid-treated cancer patients with poorly-controlled chronic pain: a randomized, placebo-controlled, graded-dose trial. J Pain, 13(5):43849. Available from: http://www.cannabis-med.org/studies/ww_en_db_study_show.php?s_id=491 .

[48] Priestman, T.J., Priestman, S.G. (1984). An initial evaluation of Nabilone in the control of radiotherapy-induced nausea and vomiting. Clin Radiol., 35(4):265-6. Available from: http://www.cannabis-med.org/studies/ww_en_db_study_show.php?s_id=237 .

[49] Sallan, S.E., Cronin, C., Zelen, M., Zinberg, N.E. (1980). Antiemetics in patients receiving chemotherapy for cancer: a randomized comparison of delta-9-tetrahydrocannabinol and prochlorperazine. New England Journal of Medicine, 302(3):135-138. Available from: http://www.cannabis-med.org/studies/ww_en_db_study_show.php?s_id=3 .

[50] Sallan, S.E., Zinberg, N.E., Frei, E. (1975). Antiemetic effect of delta-9-tetrahydrocannabinol in patients receiving cancer chemotherapy. New England Journal of Medicine, 293(16):795-797. Available from: http://www.cannabis-med.org/studies/ww_en_db_study_show.php?s_id=4 .

[51] Steele, N., Gralla, R.J., Braun, D.W. Jr, Young, C.W. (1980). Double-blind comparison of the antiemetic effects of nabilone and prochlorperazine on chemotherapy-induced emesis. Cancer Treatment Report, 64(2-3):219-24. Available from:

http://www.cannabis-med.org/studies/ww_en_db_study_show.php?s_id=131 .

[52] Strasser, F., Luftner, D., Possinger, K., Ernst, G., Ruhstaller, T., Meissner, W., Ko, Y.D., Schnelle, M., Reif, M., Cerny, T. (2006). Comparison of orally administered cannabis extract and delta-9-tetrahydrocannabinol in treating patients with cancer-related anorexia-cachexia syndrome: a multicenter, phase III, randomized, double-blind, placebo-controlled clinical trial from the Cannabisin-Cachexia-Study-Group. J Clin Oncol, 24(21):3394-400. Available from: http://www.cannabismed.org/studies/ww_en_db_study_show.php?s_id=195 . 
South American Journal of Clinical Research

Volume 3, Issue 1, 2016

[53] Ungerleider, J.T., Andrysiak, T., Fairbanks, L., Goodnight, J., Sarna, G., Jamison, K. (1982). Cannabis and cancer chemotherapy: a comparison of oral delta-9-THC and prochlorperazine. Cancer, 50:636-645. Available from:

http://www.cannabis-med.org/studies/ww_en_db_study_show.php?s_id=65 .

[54] Ungerleider JT, Sarna G, Fairbanks LA, Goodnight J, Andrysiak T, Jamison K. (1985). THC or Compazine for the cancer chemotherapy patient--the UCLA study. Part II: Patient drug preference. American Journal of Clinical Oncology, 8: 142-147. Available from: http://www.cannabismed.org/studies/ww_en_db_study_show.php?s_id=34 .

[55] Vinciguerra, V., Moore, T., Brennan E. (1988). Inhalation marijuana as an antiemetic for cancer chemotherapy. New York State Journal of Medicine, 88:525-527. Available from: http://www.cannabis-med.org/studies/ww_en_db_study_show.php?s_id=155 .

[56] Wadleigh, R., Spaulding, G.M., Lumbersky, B., Zimmer, M., Shepard, K., Plasse, T. (1990). Dronabinol enhancement of appetite in cancer patients. Proc Am Soc Oncology, 9: 331. Available from: http://www.cannabis-med.org/studies/ww_en_db_study_show.php?s_id=149 .

[57] Waissengrin, B., Urban, D., Leshem, Y., Garty, M., Wolf, I. (2015). Patterns of use of medical cannabis among Israeli cancer patients: a single institution experience. J Pain Symptom Manage, 49(2):223-30. doi: http://10.1016/j. [PubMed PMID: 24937161].

[58] Zutt, M., Hanssle, H., Emmert, S., Neumann, C., Kretschmer, L. (2006). Dronabinol for supportive therapy in patients with malignant melanoma and liver metastases. Hautarzt, 57(5):423-7. Available from: http://www.cannabis-med.org/studies/ww_en_db_study_show.php?s_id=180 . 